\title{
Pre-pregnancy weight, gestational weight gain, and the gut microbiota of mothers and their infants
}

\author{
Maggie A. Stanislawski ${ }^{1,4}$, Dana Dabelea ${ }^{1,2}$, Brandie D. Wagner ${ }^{1}$, Marci K. Sontag ${ }^{1}$, Catherine A. Lozupone ${ }^{2 \dagger}$ \\ and Merete Eggesbø $\varnothing^{3^{*}+}$
}

\begin{abstract}
Background: Recent evidence supports that the maternal gut microbiota impacts the initial infant gut microbiota. Since the gut microbiota may play a causal role in the development of obesity, it is important to understand how pre-pregnancy weight and gestational weight gain (GWG) impact the gut microbiota of mothers at the time of delivery and their infants in early life. In this study, we performed $16 \mathrm{~S}$ rRNA gene sequencing on gut microbiota samples from 169 women 4 days after delivery and from the 844 samples of their infants at six timepoints during the first 2 years of life. We categorized the women (1) according to pre-pregnancy body mass index into overweight/obese (OW/OB, BMl $\geq 25)$ or non-overweight/obese (BMI < 25) and (2) into excessive and non-excessive GWG in the subset of mothers of full-term singleton infants $(N=116)$. We compared alpha diversity and taxonomic composition of the maternal and infant samples by exposure groups. We also compared taxonomic similarity between maternal and infant gut microbiota.

Results: Maternal OW/OB was associated with lower maternal alpha diversity. Maternal pre-pregnancy OW/OB and excessive GWG were associated with taxonomic differences in the maternal gut microbiota, including taxa from the highly heritable family Christensenellaceae, the genera Lachnospira, Parabacteroides, Bifidobacterium, and Blautia. These maternal characteristics were not associated with overall differences in the infant gut microbiota over the first 2 years of life. However, the presence of specific OTUs in maternal gut microbiota at the time of delivery did significantly increase the odds of presence in the infant gut at age 4-10 days for many taxa, and these included some lean-associated taxa.
\end{abstract}

Conclusions: Our results show differences in maternal gut microbiota composition at the time of delivery by prepregnancy weight and GWG, but these changes were only associated with limited compositional differences in the early life gut microbiota of their infants. Further work is needed to determine the degree to which these maternal microbiota differences at time of birth with OW/OB and GWG may affect the health of the infant over time and by what mechanism.

\section{Background}

Obesity prevalence among children and adolescents has increased dramatically in recent years [1, 2]. Maternal obesity and excessive gestational weight gain (GWG) are associated with increased risk for offspring obesity, and these associations are not fully explained by genetic and

\footnotetext{
* Correspondence: Merete.Eggesbo@fhi.no

${ }^{\dagger}$ Equal contributors

${ }^{3}$ Department of Environmental Exposure and Epidemiology, Norwegian Institute of Public Health, PO Box 4404, Oslo, Norway

Full list of author information is available at the end of the article
}

lifestyle factors. Recent research suggests that the gut microbiota may also contribute towards the development of obesity, and it has been hypothesized that the gut microbiota may be a mechanism to explain the transgenerational transmission of obesity risk [3]. The maternal gut microbiota may influence infant obesity risk through in utero programming effects [4] or through vertical transfer of obesogenic gut microbiota from mother to child during birth [5] and in breastmilk [6]. Understanding the relationship between the gut microbiota and obesity in mothers and their children 
may offer unique opportunities to interrupt the cycle of obesity [3].

There is increasing evidence to support that the gut microbiota may play a causal role in obesity $[7,8]$. Some studies have shown differences in maternal gut microbiota at different timepoints during pregnancy by obesity status and gestational weight gain $[9,10]$. Research about whether these maternal characteristics are associated with differences in the early infant gut microbiota has been inconsistent [11-13]. However, gut microbiota during infancy has been associated with rapid early growth and later overweight and obesity $[14,15]$, and numerous exposures known to impact the early gut microbiota, such as birth via cesarean section and antibiotics, have also been associated with increased obesity risk $[16,17]$.

In this study, we evaluate whether maternal prepregnancy overweight/obese $(\mathrm{OW} / \mathrm{OB})$ or excessive gestational weight gain (GWG) are associated with differences in the maternal gut microbiota at the time of delivery or in the gut microbiota of their infants during the first 2 years of life. We also assess the similarity between maternal gut microbiota at the time of delivery and early infant gut microbiota. This is a very important area of research because the gut microbiota is alterable-through diet, pre- and probiotics, and antibiotic usage. Understanding the relationship between the gut microbiota and obesity in mothers and their infants may offer opportunities for obesity prevention measures.

\section{Methods}

\section{Study cohort}

NoMIC is a Norwegian birth cohort of 552 children designed to study the establishment of gut microbiota during infancy and its consequences for child health. Participating mothers, recruited between 2002 and 2005, were asked to fill out periodic questionnaires and to collect and freeze fecal samples from themselves at 4 days post-partum, and from their infants at days 4 , $10,30,120,365$, and 730 post-birth. Study personnel retrieved the fecal samples and kept them frozen during transport to the Biobank of the Norwegian Institute of Public Health, Oslo, where they were stored at $-20{ }^{\circ} \mathrm{C}$ upon arrival.

The study was approved by the Regional Ethics Committee for Medical Research in Norway (approval ref. 2002, S-02216) and the Norwegian Data Inspectorate (ref 2002/1934-2). The approvals, as well as informed consent from the mothers, were obtained prior to collection of data and samples. The NoMIC study was funded by the FRIMEDBIO program at the Norwegian Research Council.

\section{Study sample}

This study includes the subset of 169 mothers from NoMIC who provided a fecal sample, whose sample provided high quality Illumina data, and for whom both height and pre-pregnancy weight were available to calculate BMI (Additional file 1: Figure S1). Additionally, 844 gut microbiota samples during the first 2 years of life were available from the 181 children of these women (Additional file 1: Figure S1). This study sample showed some differences from the larger NoMIC cohort; they had lower median BMI, were more educated, and were less likely to smoke during pregnancy (Additional file 1: Table S1).

\section{Exposure definitions}

Pre-pregnancy BMI was based on maternal self-report of weight at the first clinic visit; the median time of the first visit was at 9 weeks of gestation (IQR 7.3-11.3 weeks). At that visit, height and weight were measured and information on pre-pregnancy weight was obtained from the mother. A large discrepancy within the time frame of only 9 weeks would likely have been noted by the health workers. Moreover, GWG has been validated in our study (see below). Pre-pregnancy BMI was initially categorized as underweight, normal weight, overweight, and obese according to standard definitions [18]. We then further combined these groups into the following: (1) non-OW/OB: underweight $(N=7)$ and normal weight $(N=110)$, BMI $<25(N=117)$; and (2) OW/OB: overweight $(N=32)$ and obese $(N=20)$, BMI $>25$ $(N=52)$.

When evaluating the impacts of excessive GWG on the gut microbiota, women who were missing GWG $(N=1)$ and not full term $(N=50)$ were excluded because there are not well-established weight gain recommendations for pre-term births. Furthermore, we chose to exclude mothers of twins since there were only two, making this difficult to account for in statistical models, and since the weight gain guidelines for mothers of twins are considered provisional. Thus, the sample size for the analysis of GWG was $N=116$ (Additional file 1: Figure S1). The recommended range of the Institute of Medicine (IOM) were used to define adequate GWG, which is based on pre-pregnancy BMI (Additional file 1: Table S2); weight gain less than the recommended range for the respective BMI group was considered "low" $(N=12)$, within the range as "adequate" $(N=41)$ and greater was considered "excessive" $(N=63)$ [19]. We grouped low and adequate $(N=41)$ GWG together due to the small number in the low group, and compared to excessive GWG. GWG was calculated using the prepregnancy weight and final weight from self-report in a questionnaire approximately 1-month post-delivery. When missing self-reported final weight (9\%), the final 
recorded weight preceding birth in the medical records of prenatal clinic visits was used. Self-reported GWG has been validated in the larger NoMIC study among 380 subjects with both self-reported and objective records of GWG obtained from their pregnancy journals. The Spearman correlation between the selfreported GWG and GWG according to pregnancy journals was 0.95 , with a $p$ value of $<0.001$ and the corresponding intraclass correlation (ICC) was 0.94, with a $p$ value of $<0.001$.

\section{Additional data sources}

Maternal questionnaires provided information on mode of delivery, education, parity, maternal smoking, ethnicity, and use of antibiotics. Maternal age at delivery was calculated based on birth date from the Norwegian personal identification number. We obtained information on gestational age and preterm delivery from the Medical Birth Registry of Norway.

\section{Processing of microbial samples}

DNA was extracted using standard protocols, as previously described for this cohort [20]. The extracted DNA was amplified using polymerase chain reaction (PCR) with barcoded primers targeting the V4 region of $16 \mathrm{~S}$ ribosomal RNA (rRNA). Sequences were generated using an Illumina HiSeq instrument (Illumina, San Diego, CA). Operational taxonomic units (OTUs) were assigned using UCLUST [21] as implemented in QIIME [22] via a closed reference-based system using the Greengenes 13.8 [23] database and a 97\% threshold. A rarefied OTU table at 5000 sequences per sample served as input for the analyses.

\section{Statistical analysis}

We compared maternal demographic and birth characteristics by pre-pregnancy weight group and excessive GWG status using chi-squared tests for categorical variables and Wilcoxon rank-sum tests for continuous variables.

\section{Maternal alpha diversity}

Alpha diversity measures the microbial diversity of each sample. There are many alpha diversity measures, and they differ in how they weight richness and evenness and whether they incorporate phylogenetic distance. We chose to evaluate three measures of alpha diversity: Shannon diversity index (evenly weights richness and evenness), PD whole tree (emphasizes phylogenetic diversity), and observed species (number of OTUs observed at standardized sequencing depth; richness). Rank-based regression was used to model alpha diversity measures with pre-pregnancy weight status as the primary covariate of interest. The following maternal characteristics were controlled for in the analysis: maternal age, education $(<12$ years, 12 years, $>12$ years), Norwegian ethnicity, parity (nulliparous, 1 prior child, $>1$ prior child), twins, and smoking during pregnancy (never, former, occasional, daily $\leq 10$, daily $>10$ ). The same methods were utilized for comparing women by GWG group, except that twins were excluded from the list of covariates since all births were singleton.

\section{Association between maternal characteristics and maternal microbiota composition}

We visualized relationships between microbiota diversity across samples using principal coordinate analysis (PCoA) plots of weighted and unweighted UniFrac distance [24]. Random forests were first used as a feature selection technique in order to evaluate which OTUs were most important to differentiate samples based on maternal pre-pregnancy OW/OB status [25]. This machine learning approach ranks factors in terms of their ability to discriminate exposure status, while taking into account the interrelationships in high-dimensional complex data. This method uses decision trees, and each tree is trained on a subset of the data and then tested on the remaining data; the error of these repeated tests is called the "out of bag" error rate. Breiman's random forest algorithm with down sampling [25] was used to classify women's pre-pregnancy weight group based on the percent abundance of the OTUs. All OTUs that were present in less than $10 \%$ of the samples and those with a maximum percent abundance less than $0.25 \%$ were excluded; 448 OTUs met these prevalence cutoffs. The R function varselRF [26] was used to select the most important taxa for classifying the exposure. This is a recursive technique that eliminates variables based on the importance scores. We evaluated the classification accuracy of these selected features by computing the ratio of the out of bag error from a random forest using these features to classify simulated random data to the error from a similar random forest classifying the true exposure status [27].

Since random forests do not provide information on the nature of the relationships between these exposures and the selected taxa, we assessed the direction of the associations by modeling the OTUs individually as outcomes in beta-binomial generalized linear regressions, which account for overdispersion in the sequence counts, using the SAS procedure NLMIXED. The models included the total number of sequences (5000 for all taxa) as an offset in order to allow for inference on the relative abundance. We also included covariates in the models, controlling for the same maternal characteristics as in the models of alpha diversity.

These methods were repeated for different scenarios. First, genus level taxonomies were used rather than 
OTUs to model pre-pregnancy weight group with the same prevalence cutoffs as for OTUs, leaving 169 genera. We used these same methods to model excess GWG in the subset of women who were full term with singleton births, both at the OTU level and the genus level of taxonomy. The random forest of OTUs included the 403 OTUs that met the prevalence cutoffs; the random forest at the genus level included 80 genera.

We also performed sensitivity analyses. First, we examined continuous exposures of maternal pre-pregnancy BMI rather than OW/OB, and GWG $(\mathrm{kg})$ in excess of the recommended GWG range rather than the dichotomous measure. Both of these showed fairly consistent taxonomic associations with the gut microbiota. We also excluded overweight women, comparing $\mathrm{OB}$ to nonoverweight/obese. However, the classification accuracy was worse than in the primary analysis.

\section{Association between maternal characteristics and infant microbiota alpha diversity and composition over the first 2 years of life}

In order to evaluate the association between exposure to maternal OW/OB or excessive GWG and infant gut alpha diversity, we used longitudinal hierarchical linear regressions with a random intercept for infant within family. We controlled for the same maternal characteristics as in the models of maternal alpha diversity, in addition to the following potential mediating variables: delivery mode at birth (cesarean section or vaginal), gestational age at birth, exclusive breastfeeding (yes/no at the time of the sample), and antibiotic exposure (yes/no at the time of the sample).

Random forests were used to identify which OTUs in infant gut microbiota samples over the first 2 years of life were most important to differentiate samples based on (1) maternal OW/OB and (2) excessive GWG [25]. The same prevalence thresholds and methods were used as in the random forests of maternal gut microbiota samples, as described above. In addition to the OTUs, we included sampling time as a predictor, to allow for interactions between taxonomic abundance and age. The analysis of maternal OW/OB included 253 OTUS; the analysis of GWG included 251 OTUs.

\section{Comparison of maternal and early infant gut microbiota}

To compare maternal gut microbiota taxa at the time of delivery with that of the infant at days 4 and 10, we used longitudinal binary logistic regression with a random intercept by child. OTU-level presence/absence of maternal samples was used as the exposure with infant presence/absence as the outcome for each of the OTUs selected to differentiate maternal $\mathrm{OW} / \mathrm{OB}$ status or excessive GWG. A similar logistic model was used to evaluate presence/absence across all OTUs together, as well as across all of OTUs selected to differentiate the maternal exposures, in order to assess the overall maternal-infant taxonomic association.

We used SAS v9.4 (SAS Institute Inc., Cary, North Carolina), R v3.2.0 [28], and QIIME v1.9.0 [22] for analyses. $p$ values less than 0.05 were considered statistically significant.

\section{Results}

Women with and without excess pre-pregnancy weight generally had similar characteristics (Table 1). Excess weight women had slightly less education and were more likely to be smokers, as were women with excessive GWG (Additional file 1: Table S3). There was a higher proportion of women who were overweight prepregnancy among those with excessive GWG relative to those with non-excessive GWG.

\section{Maternal alpha diversity}

The median alpha diversity was significantly lower among OW/OB women for all three alpha diversity measures examined (Table 1). In regression models controlling for maternal age, education, Norwegian ethnicity, parity, twins, and smoking during pregnancy (Table 2), Shannon diversity and PD whole tree remained significantly lower among OW/OB women. Controlling for gestational age and maternal antibiotic use during pregnancy did not substantially alter the results.

In the subset of full term women, excessive GWG was not associated with significant differences in alpha diversity (Shannon: $\beta=-0.1,95 \% \mathrm{CI}-0.3,0.1 ; p$ value $=0.53$; PD: $\beta=-0.1,95 \% \mathrm{CI}-1.9,1.8$; $p$ value $=0.96$; observed species: $\beta=-4.0,95 \% \mathrm{CI}-28.3,20.2 ; p$ value $=0.75$ ). Controlling for maternal BMI, gestational age and maternal antibiotic usage did not alter the null results.

\section{Maternal microbiota composition}

The overall microbiota composition of the maternal samples resembled that typical of healthy European/US adults, with a dominance of taxa from the phyla of Firmicutes, Bacteroidetes, and Actinobacteria, and infant samples became more similar to maternal samples with age (Additional file 1: Figure S2) [29, 30]. Principal coordinate analysis plots of UniFrac distance also showed a progression of infant microbiota to one resembling maternal (adult-like) compositions over the first year of life, but did not show strong differentiation between gut microbiota samples by maternal OW/OB or excessive GWG status (Additional file 1: Figure S3).

Gut microbiota composition showed numerous differences by pre-pregnancy weight group. Using the most important OTUs from the random forest to classify prepregnancy weight status, the out of bag error rate was 
Table 1 Demographic and birth characteristics of mothers by pre-pregnancy weight group: overweight/obese (OW/OB) and non-OW/OB

\begin{tabular}{|c|c|c|c|c|}
\hline \multirow[t]{2}{*}{ Variable } & Total $(N=169)$ & Non-OW/OB $(N=117)$ & OW/OB $(N=52)$ & $p$ value \\
\hline & \multicolumn{3}{|l|}{$N(\%)$ or median (IQR) } & \\
\hline$\overline{B M I}$ & $23.1(21.1-25.5)$ & $21.6(20.2-23.1)$ & $27.2(25.7-31.7)$ & $<.001$ \\
\hline \multicolumn{5}{|l|}{ Pre-pregnancy BMI category } \\
\hline Underweight & $7(4.1 \%)$ & $7(6.0 \%)$ & $0(0.0 \%)$ & \multirow[t]{4}{*}{$<.001$} \\
\hline Normal & $110(65.1 \%)$ & $110(94.0 \%)$ & $0(0.0 \%)$ & \\
\hline Overweight & $32(18.9 \%)$ & $0(0.0 \%)$ & $32(61.5 \%)$ & \\
\hline Obese & $20(11.8 \%)$ & $0(0.0 \%)$ & $20(38.5 \%)$ & \\
\hline Ethnic Norwegian & $142(87.1 \%)$ & 95 (84.1\%) & 47 (94.0\%) & 0.13 \\
\hline \multicolumn{5}{|l|}{ Maternal education } \\
\hline$<12$ years education & $9(5.6 \%)$ & $4(3.6 \%)$ & $5(10.4 \%)$ & \multirow[t]{3}{*}{0.01} \\
\hline 12 years education & $28(17.5 \%)$ & 19 (17.0\%) & $9(18.8 \%)$ & \\
\hline$>12$ years education & $123(76.9 \%)$ & $89(79.5 \%)$ & $34(70.8 \%)$ & \\
\hline Maternal age (years) & $30.0(27.0-34.0)$ & $30.0(27.0-33.0)$ & $30.0(27.5-34.0)$ & 0.90 \\
\hline \multicolumn{5}{|l|}{ Parity } \\
\hline No prior pregnancies & $83(49.1 \%)$ & $57(48.7 \%)$ & $26(50.0 \%)$ & \multirow[t]{3}{*}{0.62} \\
\hline 1 prior child & $53(31.4 \%)$ & 39 (33.3\%) & $14(26.9 \%)$ & \\
\hline$>1$ prior child & $33(19.5 \%)$ & $21(17.9 \%)$ & $12(23.1 \%)$ & \\
\hline Twins & $11(6.5 \%)$ & $8(6.8 \%)$ & $3(5.8 \%)$ & 1.00 \\
\hline \multicolumn{5}{|c|}{ Maternal smoking at beginning of pregnancy } \\
\hline Never smoker & $111(66.5 \%)$ & $77(67.0 \%)$ & $34(65.4 \%)$ & \multirow[t]{5}{*}{0.01} \\
\hline Past smoker & $41(24.6 \%)$ & $28(24.3 \%)$ & $13(25.0 \%)$ & \\
\hline Occasional & $7(4.2 \%)$ & $5(4.3 \%)$ & $2(3.8 \%)$ & \\
\hline Daily smoker $\leq 10$ & $4(2.4 \%)$ & $2(1.7 \%)$ & $2(3.8 \%)$ & \\
\hline Daily smoker $>10$ & $4(2.4 \%)$ & $3(2.6 \%)$ & $1(1.9 \%)$ & \\
\hline \multicolumn{5}{|l|}{ Diabetes } \\
\hline No diabetes & $167(98.8 \%)$ & $116(99.1 \%)$ & $51(98.1 \%)$ & \multirow[t]{3}{*}{0.21} \\
\hline $\mathrm{T} 1$ & $1(0.6 \%)$ & $1(0.9 \%)$ & $0(0.0 \%)$ & \\
\hline $\mathrm{T} 2$ & $1(0.6 \%)$ & $0(0.0 \%)$ & $1(1.9 \%)$ & \\
\hline Glucose in urine & $15(8.9 \%)$ & $8(6.8 \%)$ & $7(13.5 \%)$ & 0.16 \\
\hline High BP & $8(4.7 \%)$ & $4(3.4 \%)$ & $4(7.7 \%)$ & 0.25 \\
\hline Gestational weight gain $(\mathrm{kg})$ & $14.0(10.0-18.0)$ & $15.0(12.0-19.0)$ & $11.0(8.1-15.0)$ & $<.001$ \\
\hline \multicolumn{5}{|c|}{ GWG relative to IOM recommendations } \\
\hline Low & $12(10.3 \%)$ & $12(14.6 \%)$ & $0(0.0 \%)$ & \multirow[t]{3}{*}{0.06} \\
\hline Adequate & $41(35.3 \%)$ & $27(32.9 \%)$ & $14(41.2 \%)$ & \\
\hline Excessive & $63(54.3 \%)$ & $43(52.4 \%)$ & $20(58.8 \%)$ & \\
\hline Gestational age (days) & $278(254-285)$ & $279(256-285)$ & $277(253-287)$ & 0.80 \\
\hline Birth weight (kg) & $3.37(2.46-3.75)$ & $3.31(2.47-3.69)$ & $3.44(2.46-3.84)$ & 0.29 \\
\hline C-section & $50(29.6 \%)$ & $31(26.5 \%)$ & 19 (36.5\%) & 0.19 \\
\hline Maternal antibiotics & $52(32.1 \%)$ & $35(31.0 \%)$ & $17(34.7 \%)$ & 0.71 \\
\hline Day of birth & $9(5.6 \%)$ & $6(5.3 \%)$ & $3(6.1 \%)$ & 1.00 \\
\hline Day before birth & $2(1.2 \%)$ & $1(0.9 \%)$ & $1(2.0 \%)$ & 0.51 \\
\hline Week before birth & $6(3.7 \%)$ & $2(1.8 \%)$ & $4(8.2 \%)$ & 0.07 \\
\hline Month before birth & $9(5.6 \%)$ & $6(5.3 \%)$ & $3(6.1 \%)$ & 1.00 \\
\hline
\end{tabular}


Table 1 Demographic and birth characteristics of mothers by pre-pregnancy weight group: overweight/obese (OW/OB) and non-OW/OB (Continued)

\begin{tabular}{llll}
\hline \multicolumn{1}{c}{ 1 month before birth } & $26(16.0 \%)$ & $18(15.9 \%)$ & $8(16.3 \%)$ \\
Antibiotics given to newborn & $14(8.6 \%)$ & $7(6.2 \%)$ & $7(14.3 \%)$ \\
Alpha diversity & & & 1.00 \\
$\quad$ Shannon & $5.7(5.3-6.1)$ & $5.7(5.4-6.1)$ & $5.5(5.0-5.9)$ \\
Observed species & $365(307-400)$ & $375(315-400)$ & $338(292.5-385)$ \\
PD whole tree & $27.3(23.6-30.1)$ & $28.5(24.0-30.6)$ & $25.9(21.8-29.4)$ \\
\hline
\end{tabular}

18.9\%; this classification accuracy was 2.4 times better than completely random guessing.

The most important OTUs from the random forest included members of the genera Bifidobacterium and Parabacteroides, as well as nine members of the order Clostridiales. These taxa were modeled using betabinomial regression models (Fig. 1; Additional file 1: Table S4). Eight of these OTUs were significantly different by maternal pre-pregnancy weight group when adjusting for maternal characteristics.

The random forest using genus level taxa to predict pre-pregnancy weight status had an out of bag error rate of $28.4 \%$; the predictive accuracy was 1.9 times as good as random guessing. Figure 1 shows the distributions of the genus-level taxa ranked as most important in the random forest and modeled using beta-binomial regressions (Additional file 1: Table S5), which were generally consistent with the OTU-level analysis.

The random forest to classify GWG group based on OTUs had an out of bag error rate of $24.1 \%$; this classification accuracy was twice as accurate as random guessing. The most important taxa included members of the genera Methanobrevibacter, Bifidobacterium, and Bacteroides, as well as seven OTUs of the order Clostridiales. In the betabinomial regression models, there were three OTUs that were significantly higher among women with excess
GWG when controlling for maternal characteristics (Fig. 2; Additional file 1: Table S6). The random forest using genus-level taxa as predictors had an out of bag error rate of $31.9 \%$, which was 1.7 times as good as random guessing, and showed consistent patterns as the OTU level analysis (Fig. 2; Additional file 1: Table S7).

\section{Association between maternal characteristics and infant microbiota alpha diversity and composition over the first 2 years of life}

The alpha diversity of infant gut microbiota over the first 2 years of life did not show an association with exposure to maternal OW/OB or excessive GWG in any of the examined measures of alpha diversity (Additional file 1: Table S8). Random forests trained to classify the infant gut microbiota according to these maternal characteristics had poor accuracy (Additional file 1: Tables S9-S10), being only $22 \%$ better than random guessing for classifying taxa by maternal OW/OB and $8 \%$ better for classifying excessive GWG. This low level of accuracy suggests that the models could not identify clinically meaningful taxonomic differences [31]. We also investigated whether other factors that have been previously associated with differences in the infant gut microbiota were likewise associated in this cohort, including mode of delivery, breastfeeding, and antibiotic exposure using a permutational ANOVA of

Table 2 Results of rank-based regression models of alpha diversity of maternal gut microbiota samples at the time of delivery

\begin{tabular}{|c|c|c|c|c|c|c|}
\hline & Shannon & & PD & & Observed species & \\
\hline & $\beta(95 \% \mathrm{Cl})$ & $p$ value & $\beta(95 \% \mathrm{Cl})$ & $p$ value & $\beta(95 \% \mathrm{Cl})$ & $p$ value \\
\hline Intercept & $5.82(5.01,6.62)$ & $<0.01$ & $28.09(21.03,35.15)$ & $<0.01$ & $353.41(262.22,444.6)$ & $<0.01$ \\
\hline Maternal OW/OB & $-0.19(-0.38,-0.01)$ & 0.04 & $-1.74(-3.36,-0.12)$ & 0.04 & $-19.85(-40.52,0.83)$ & 0.06 \\
\hline Maternal age & $0.02(-0.01,0.04)$ & 0.14 & $0.09(-0.12,0.29)$ & 0.40 & $1.79(-0.82,4.41)$ & 0.18 \\
\hline Norwegian & $-0.27(-0.53,-0.01)$ & 0.04 & $-1.45(-3.73,0.82)$ & 0.21 & $-32.73(-61.78,-3.69)$ & 0.03 \\
\hline Education & $-0.11(-0.27,0.05)$ & 0.20 & $-0.33(-1.74,1.07)$ & 0.64 & $-1.22(-19.16,16.72)$ & 0.89 \\
\hline Parity: $>1$ prior child & $-0.1(-0.37,0.17)$ & 0.48 & $-0.38(-2.78,2.02)$ & 0.76 & $-10.59(-41.26,20.08)$ & 0.50 \\
\hline Parity: 1 prior child & $-0.15(-0.34,0.05)$ & 0.14 & $-1.46(-3.18,0.26)$ & 0.10 & $-24.25(-46.19,-2.31)$ & 0.03 \\
\hline Twins & $-0.18(-0.53,0.16)$ & 0.31 & $-1.54(-4.59,1.5)$ & 0.32 & $-16.01(-54.94,22.91)$ & 0.42 \\
\hline Maternal smoking & $-0.07(-0.17,0.02)$ & 0.15 & $-0.54(-1.41,0.32)$ & 0.22 & $-6.08(-17.14,4.98)$ & 0.28 \\
\hline
\end{tabular}

$\beta$ indicates the parameter estimate and $95 \% \mathrm{Cl}$ is the $95 \%$ confidence interval 


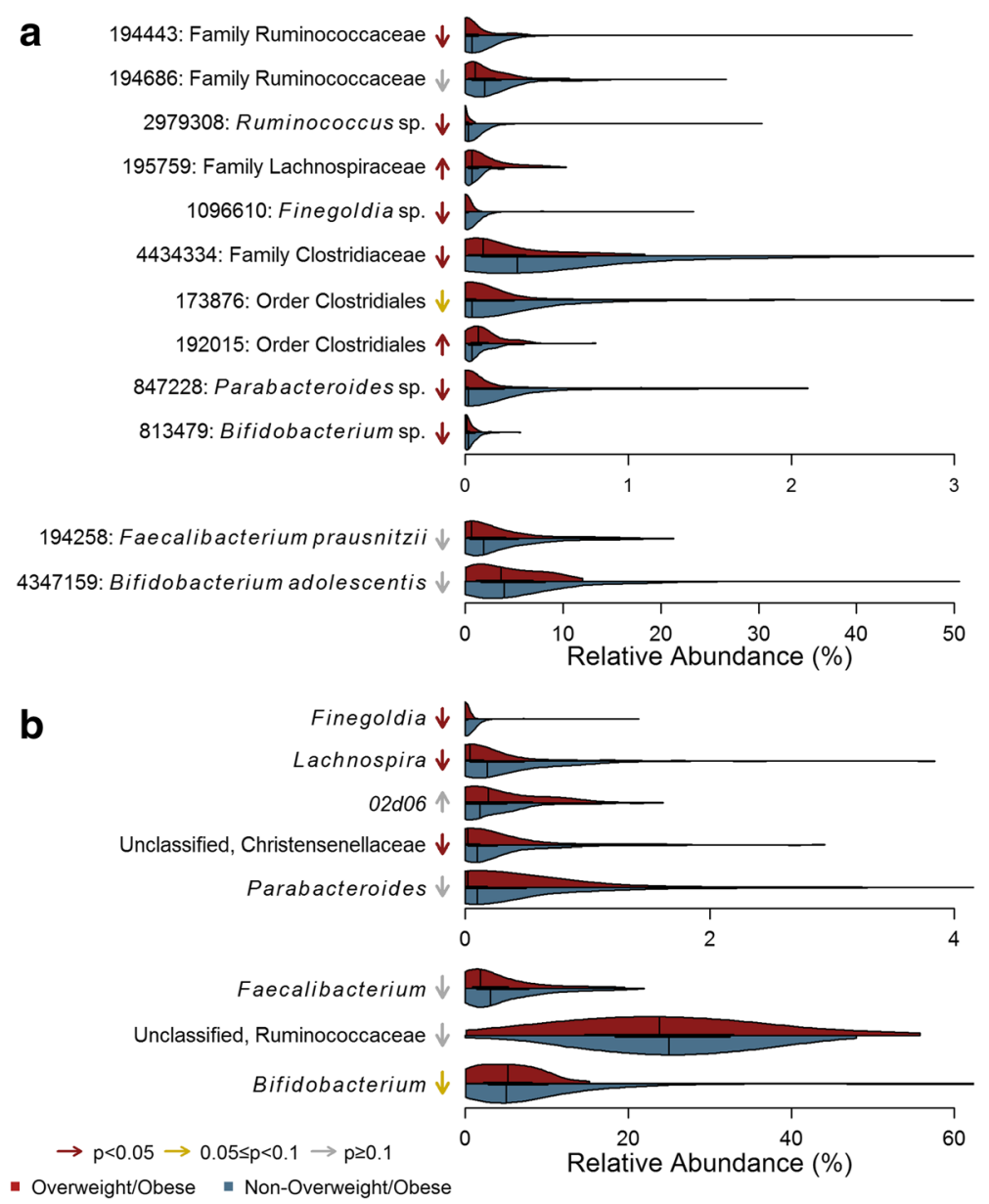

Fig. 1 Violin plots of important $\mathbf{a}$ OTUs and $\mathbf{b}$ genera from Random Forests for classifying maternal OW/OB status. Arrows indicate the direction of association with maternal $O W / O B$, with color corresponding to degree of significance of uncorrected $p$ values in beta-binomial regression models controlling for the following maternal characteristics: maternal age, maternal education, Norwegian ethnicity, twins, maternal smoking, and parity

unweighted and weighted UniFrac distance. All of these exposures were highly significantly associated ( $p$ values $\leq 0.002)$ with both the phylogeny (unweighted UniFrac) and abundance (weighted UniFrac) of the infant gut microbiota.

\section{Comparison of maternal and early infant gut microbiota}

The presence of OTUs in maternal gut microbiota at the time of delivery significantly increased the odds of presence in the early infant gut microbiota; this was true when evaluating the average association across all OTUs $(\mathrm{OR}=2.8,95 \% \mathrm{CI} 2.8,2.9 ; p$ value $<0.01)$ and when specifically evaluating those associated with maternal OW/ OB (Fig. 3; OR $=2.4,95 \%$ CI 2.0, 3.0; $p$ value $<0.001$ ). Yet, most of the individual OTUs associated with maternal OW/OB were not significantly associated between maternal-infant samples, and some were almost entirely absent from the infants. However, the presence of leanassociated OTUs in the Parabacteroides and Finegoldia genera in maternal samples were associated with increased odds of presence in the infant $(\mathrm{OR}=2.5,95 \% \mathrm{CI}$ $1,6.3 ; p$ value $<0.05$; $\mathrm{OR}=3,2,95 \%$ CI $1.5,7.1 p$ value $<0.01$, respectively). The group of OTUs highlighted as important to classify maternal excessive GWG were not significantly associated overall between maternal and infant samples (Fig. 3; OR $=1.2,95 \%$ CI 0.8, 1.6; $p$ value $=0.42$.

\section{Discussion}

Both maternal OW/OB and excessive GWG have detrimental short- and long-term health consequences for the infant, such as increased risk for fetal macrosomia, obesity, metabolic syndrome, and even all-cause mortality [3]. It is possible that the maternal gut microbiota may mediate some of the increased disease risk associated with these exposures, particularly with respect to obesity. Our results showing numerous differences in maternal gut microbiota associated with pre-pregnancy 


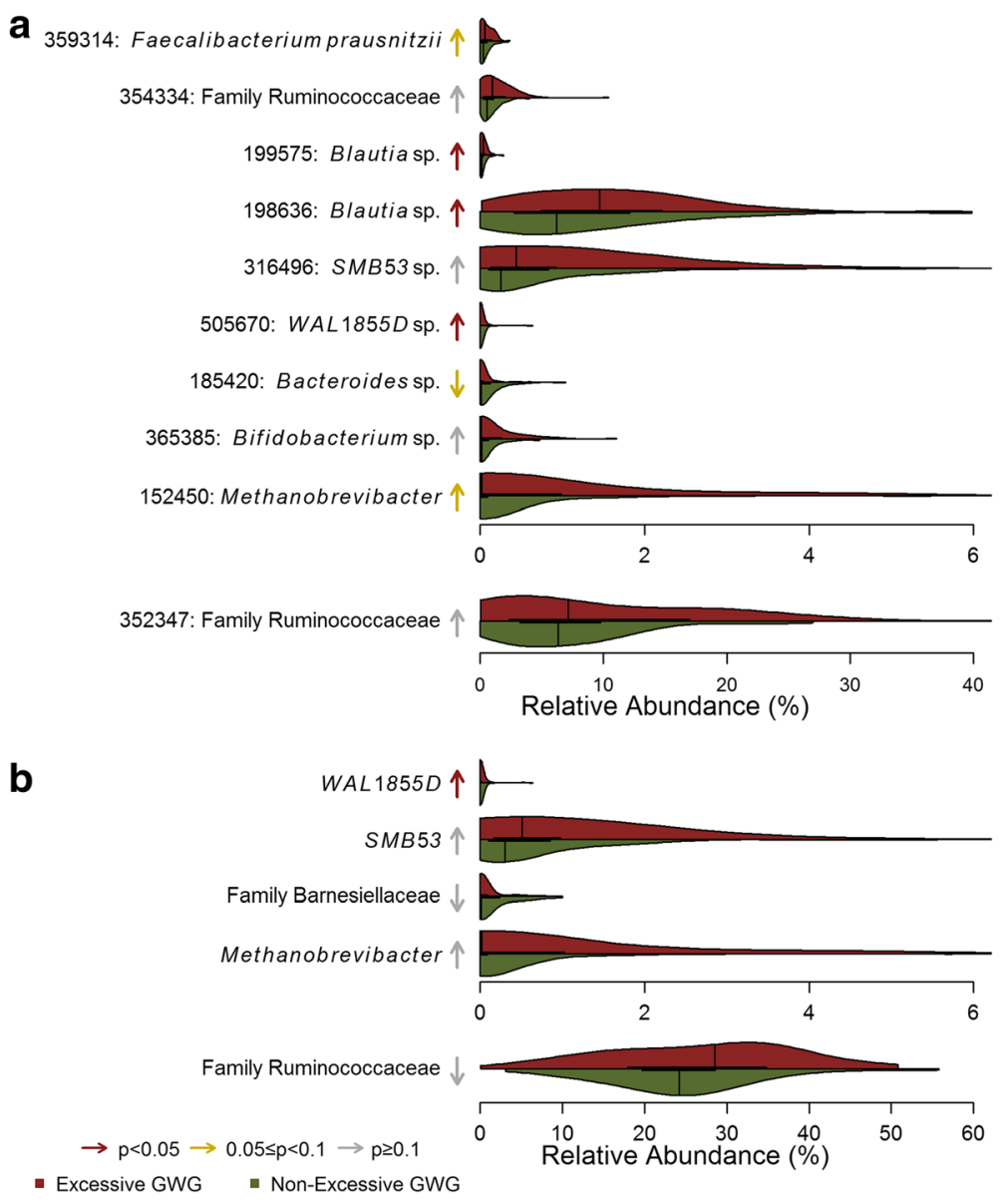

Fig. 2 Violin plots of important $\mathbf{a}$ OTUs and $\mathbf{b}$ genera from Random Forests for classifying maternal excessive GWG status. Arrows indicate the direction of association with maternal excessive GWG, with color corresponding to degree of significance of uncorrected $p$ values in beta-binomial regression models controlling for the following maternal characteristics: maternal age, maternal education, Norwegian ethnicity, maternal smoking, and parity

OW/OB and GWG lend support to this notion. We found that pre-pregnancy $\mathrm{OW} / \mathrm{OB}$ is associated with both lower maternal alpha diversity as well as differences in microbial composition, and that excess GWG is associated with compositional differences. While we might expect that these differences in maternal gut microbiota would translate into overall differences in their infants' gut microbiota, we did not find that these maternal characteristics were associated with significant differences in the infant gut microbiota over the first 2 years of life. However, we did find significant correlation between presence of taxa in the maternal and infant samples, particularly for the maternal taxa associated with pre-pregnancy weight.

Most of the taxa that differentiated maternal OW/OB in our study were higher among lean women, and many of these have shown consistent associations with leanness in prior studies, such as Parabacteroides [32, 33], Lachnospira [34], Faecalibacterium prausnitzii [35], members of the family Christensenellaceae [36], Ruminococcus [37], and Bifidobacterium [38]. Furthermore, some of these taxa may be of particular importance in the early infant gut. Abundance of Lachnospira [33, 34, 39-41] and Faecalibacterium in the first 3 months of life have been associated with risk for developing asthma [42]. Furthermore, members of Christensenellaceae family are among most heritable taxa and have shown a protective effect against weight gain in mouse studies involving fecal-transplants from obese humans [36], which could make it of key importance to explain the obesity associations across generations, as well as a key target for microbiota-based interventions.

Our results show an association between maternal $\mathrm{OW} / \mathrm{OB}$ and low alpha diversity, which has also been associated with obesity in some prior studies $[39,43]$ as well as many other diseases (including inflammatory bowel disease [35], autism [44], asthma/allergy [40], and dyslipidemia [43]) but may be a consequence of disease, 


\section{a}

194258: Faecalibacterium prausnitzii

194443: Family Ruminococcaceae

194686: Family Ruminococcaceae

2979308: Ruminococcus sp.

195759: Family Lachnospiraceae

1096610: Finegoldia sp.

4434334: Family Clostridiaceae

173876: Order Clostridiales

192015: Order Clostridiales

847228: Parabacteroides sp.

4347159: Bifidobacterium adolescentis

813479: Bifidobacterium sp.

All selected OTUs to differentiate maternal OW/OB

\section{b}

359314: Faecalibacterium prausnitzii

352347: Family Ruminococcaceae

354334: Family Ruminococcaceae

199575: Blautiasp.

198636: Blautia sp.

316496: SMB53 sp.

505670: WAL 1855D sp.

185420: Bacteroidessp.

365385: Bifidobacterium sp.

152450: Methanobrevibacter

All selected OTUs to differentiate excessive GWG

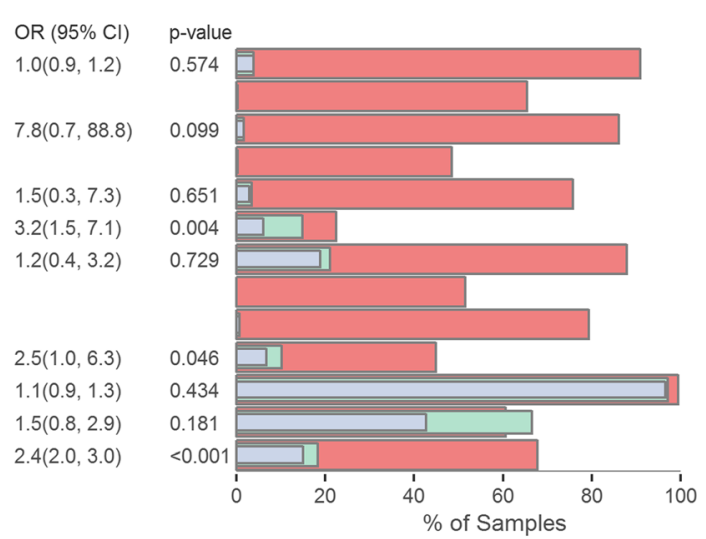

OR $(95 \% \mathrm{Cl})$

$p$-value

$1.0(1.0,1.1)$

$1.2(0.7,2.0)$

$1.1(1.0,1.3)$

$0.2(0.0,2.4)$

$2.3(0.5,10.0)$

$0.8(0.2,2.6)$

$1.2(0.8,1.6)$

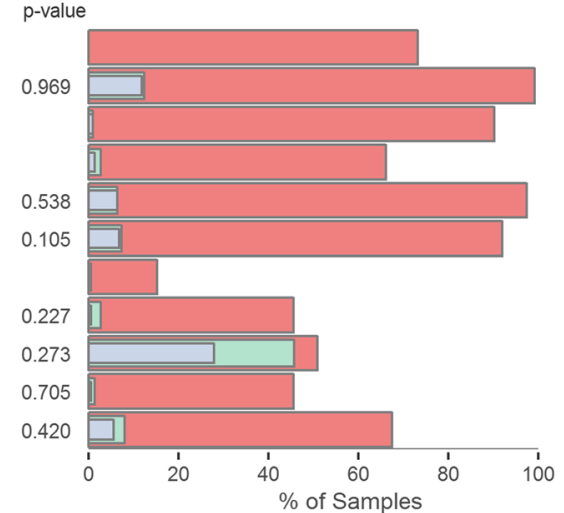

$\square$ Maternal $\quad$ Infant $\square$ Both Maternal \& Infant

Fig. 3 Plots of the presence of OTUs that differentiate a pre-pregnancy maternal OW/OB status and $\mathbf{b}$ excessive GWG in maternal and infant gut microbiota samples. The percent of samples with non-zero abundance from the mothers at the time of delivery are shown in red, and the percent of such samples from the infants at days 4-10 of life are shown in green, and from both mother and child are shown in gray for each OTU, as well as the average across these OTUs. The results of logistic regressions estimating the odds of presence in the infant based on presence in the mother are also shown. OR indicates the odds ratio, and $95 \% \mathrm{Cl}$ is the $95 \%$ confidence interval. In some cases, there was not enough data or enough variation in the presence of the OTUs across the samples to estimate the regression parameters

as longitudinal studies are limited. Koren et al. [41] showed a decrease in alpha diversity from the first to third trimester in pregnant women, although not replicated in another study of pregnant women by DiGuilo et al. [45]

The taxa associated with excessive GWG in our results are generally distinct from those associated with prepregnancy $\mathrm{OW} / \mathrm{OB}$, and show less consistent patterns with prior studies of obesity. One exception is the taxa Blautia, which was enriched with excessive GWG and has been associated with type 2 diabetes [46], and some species of this genus have been associated with obesity in a Japanese population [47]. However, other research has suggested that Blautia may also be beneficial to health in some contexts [48]. The taxa that differentiated maternal excessive GWG also tended to be absent or at non-detectible levels in most of their infants at days 410 ; they tend to be later colonizers of the infant gut.

We found that maternal OW/OB and excessive GWG were not significant determinants of the infant gut microbiota composition during the first 2 years of life.
This is not entirely surprising; infant gut microbiota changes drastically over the first few years of life, differs substantially across infants, and is affected strongly by other factors, including mode of delivery, breastfeeding, and antibiotic usage $[5,49]$. Other studies have also examined these associations, and the results have been inconsistent. Collado et al. found numerous differences according to maternal pre-pregnancy weight and gestational weight gain (GWG) in infant samples at 1 and 6 months [50]; Mueller et al. found that microbiota from the first infant stool differed by maternal OW/OB only among vaginally born infants [11]; Galley et al. found differences at 18-27 months only among infants of high socioeconomic status [13]; and Laursen et al. found no impact of maternal obesity on microbial diversity or taxonomic composition at 9 or 18 months [12]. This prior research combined with our results suggest that maternal pre-pregnancy weight and GWG are not major determinants of the overall taxonomic composition of the infant gut. However, it is possible that these maternal characteristics may influence specific infant gut microbes 
that affect later obesity risk. It is also possible that the effect of these characteristics is more pronounced among infants with the most exposure to maternal gut microbiota-e.g., those who are vaginally born, breastfed, and unexposed to antibiotics in early life. We aim to explore this hypothesis in future work in the full cohort of NoMIC infants.

The differences noted in the maternal microbiota could potentially influence the offspring through various means, including vertical transfer during and after birth which then shapes the gut colonization process of the infant. Work by Backhed et al. [5] showed that the maternal gut microbiota is a major determinant of the infant gut microbiota; $72 \%$ of early colonizers in the infant gut matched species found in maternal samples for vaginally born infants, and $41 \%$ in infants born via Csection. Similarly, we see that the presence of taxa in the maternal gut microbiota at the time of delivery is highly predictive of presence in the infant in early life. A recent study by Nayfach et al. [51] performed a strain-level analysis to further explore the variation in maternal-infant vertical transmission across taxa and over the first year of life. They found evidence of extensive vertical transmission of gut microbiota shortly after birth, particularly for certain species, including Parabacteroides distasonis and Bifidobacterium adolescentis, and that the vast majority of strains in the infants at 4 days that were transmitted from the mother persisted in the infants at 4 and 12 months. This is interesting in the context of our results because species in the Parabacteroides and Bifidobacterium genera were both associated with leanness in mothers, and presence of these taxa in the mother was predictive of presence in their infants.

Some of the taxa associated with maternal prepregnancy $\mathrm{OW} / \mathrm{OB}$ or excessive GWG tend to be later colonizing bacteria, such as Blautia, Ruminococcus, and Faecalibacterium, and they may not be transferred from the mother. However, Nayfach's work also showed that while strain-level similarity between mothers and infants significantly decreased over the first year of life, the maternal-infant species-level composition converged over time [51]. Thus, while these taxa may not be vertically transmitted to the infants, their presence in the mother may still be predictive of species-level composition in their infants at later timepoints due to shared environmental exposures, and may still impact the infant risk for obesity.

The differences in the maternal microbiota seen in our results could also have in utero impacts. The fetal programming hypothesis sets forth the notion that exposures in utero, at birth and in early life may have longterm effects on adult health [52]. A recent study by Agüero et al. [4] provides compelling evidence that in utero programming occurs, in part, through the maternal gut microbiota. Using a murine model, they found that transient maternal colonization during pregnancy had long-term effects on the innate immune system of the offspring, as well as the expression of numerous genes in the newborn intestine, including genes involved in metabolism and oxidative stress.

This study has both important strengths and limitations. Our population is much larger than many of the prior studies of maternal weight and the gut microbiota, and it includes gut microbiota data from both the mothers at the time of delivery and their infants over the first 2 years of life. The maternal vaginal and skin microbiota may play important roles in seeding the infant microbiome [53], but only gut microbiota samples were collected in this study. The population is largely of Norwegian ethnicity, which means that some of the taxonomic findings may not reflect patterns in other ethnic or racial groups, or in other geographic regions. However, the results should be internally consistent in terms of showing support for the notion that maternal weight characteristics may influence maternal gut microbiota at the time of delivery, which may affect some of the early infant taxa. Methodologically, we used machine learning techniques, which are particularly suited to the analysis of complex gut microbiota data [27], and regression models that controlled for many of the known confounders of the relationship between maternal weight and the gut microbiota. However, it is difficult to completely control for certain effects, particularly for the infant samples, which are known to be largely influenced by breastfeeding, mode of delivery, and anti-biotic exposure [5, 49].

Many of the gut microbiota associated with OW/OB in pregnant women in this study have been previously associated with obesity in non-pregnant adults. Some of the taxonomic differences noted, while not previously associated with obesity, have been associated with childhood risk for other conditions, such as asthma. Thus, pre/probiotics targeted towards obesity in the general population may be beneficial for pregnant women as well; however, there may be additional microbiota that are particularly advantageous for pregnant women around the time of delivery. For example, bacteria from the genera of Parabacteroides or Bifidobacterium, or those from the family Christensenellacea, may play a protective role against excessive weight gain and be highly heritable. These and other gut microbiota highlighted in this study offer insight into the etiology of childhood obesity and may inform future studies related to obesity prevention efforts based on the gut microbiota.

\section{Conclusions}

In this study, we found that maternal $\mathrm{OW} / \mathrm{OB}$ and excessive GWG were associated with differences in the 
maternal gut microbiota at the time of delivery. These changes were only associated with limited compositional differences in their infants. The differences seen in maternal gut microbiota could have health consequences for the child through programming effects or direct seeding of the infant gut microbiota. However, further research is needed to understand whether the maternal or infant gut microbiota are key mechanisms for the transgenerational transmission of obesity risk.

\section{Additional file}

Additional file 1: Supplemental tables and figures. (PDF $1411 \mathrm{~kb}$ )

\section{Abbreviations}

GWG: Gestational weight gain; OTU: Operational taxonomic unit; OW/ OB: Overweight/obese

\section{Acknowledgements}

The data were prepared with the help of Rob Knight, Jose Clemente, Cecilie Dahl, and Nina Iszatt.

\section{Funding}

The research leading to these results received funding from the FRIMEDBIO program at the Norwegian Research Council, grant agreement no 214324/ F20 and the European Union's Seventh Framework Programme (FP7/20072013), project EarlyNutrition under grant agreement $n^{\circ} 289,346$.

\section{Availability of data and materials}

Microbiome data and basic meta data such as gender and age will be made available on the EBI Metagenomics website [54]. Due to Norwegian laws protecting the rights of participants to have their data deleted upon request, some of the metadata used in this analysis must be requested from the authors and will be made available after a data transfer agreement has been signed.

\section{Authors' contributions}

MAS performed the analyses and wrote the manuscript. DD offered guidance on the research question and methodology. BW assisted with the statistical methodology. MKS offered feedback on the research question and the manuscript. CL guided the methodology for the analysis and interpretation of the microbiome data. ME designed and implemented the NoMIC study and guided the methodology and interpretation of the results. All authors read and approved the final manuscript.

\section{Ethics approval and consent to participate}

The study was approved by the Regional Ethics Committee for Medical Research in Norway (approval ref. 2002, S-02216) and the Norwegian Data Inspectorate (ref 2002/1934-2). The approvals, as well as informed consent from the mothers, were obtained prior to collection of data and samples.

\section{Consent for publication}

N/A.

\section{Competing interests}

The authors declare that they have no competing interests.

\section{Publisher's Note}

Springer Nature remains neutral with regard to jurisdictional claims in published maps and institutional affiliations.

\section{Author details}

${ }^{1}$ Colorado School of Public Health, Colorado, Aurora, USA. ${ }^{2}$ University of Colorado School of Medicine, Colorado, Aurora, USA. ${ }^{3}$ Department of Environmental Exposure and Epidemiology, Norwegian Institute of Public Health, PO Box 4404, Oslo, Norway. ${ }^{4}$ VA Eastern Colorado Health Care System, Denver, CO, USA.
Received: 13 June 2017 Accepted: 23 August 2017

Published online: 04 September 2017

\section{References}

1. Obesity and Overweight for Professionals: Childhood: Data | DNPAO |CDC [Internet]. [cited 2014 Apr 23]. Available from: http://www.cdc.gov/obesity/ data/childhood.html.

2. Wang $Y$, Lim H. The global childhood obesity epidemic and the association between socio-economic status and childhood obesity. Int Rev Psychiatry. 2012;24:176-88

3. Gohir W, Ratcliffe EM, Sloboda DM. Of the bugs that shape us: maternal obesity, the gut microbiome, and long-term disease risk. Pediatr Res. 2015; 77:196-204.

4. Agüero MG de, Ganal-Vonarburg SC, Fuhrer T, Rupp S, Uchimura Y, Li H, et al. The maternal microbiota drives early postnatal innate immune development. Science 2016;351:1296-1302.

5. Bäckhed F, Roswall J, Peng Y, Feng Q, Jia H, Kovatcheva-Datchary P, et al. Dynamics and Stabilization of the Human Gut Microbiome during the First Year of Life. Cell Host Microbe. 2015;17:690-703.

6. Perez PF, Doré J, Leclerc M, Levenez F, Benyacoub J, Serrant $\mathrm{P}$, et al. Bacterial Imprinting of the Neonatal Immune System: Lessons From Maternal Cells? Pediatrics. 2007:119:e724-32.

7. Harley ITW, Karp CL. Obesity and the gut microbiome: Striving for causality. Mol Metab. 2012;1:21-31.

8. Zhao L. The gut microbiota and obesity: from correlation to causality. Nat Rev Microbiol. 2013;11:639-47.

9. Dominguez-Bello MG, Costello EK, Contreras M, Magris M, Hidalgo G, Fierer $\mathrm{N}$, et al. Delivery mode shapes the acquisition and structure of the initial microbiota across multiple body habitats in newborns. Proc Natl Acad Sci. 2010;107:11971-5.

10. Makino H, Kushiro A, Ishikawa E, Muylaert D, Kubota H, Sakai T, et al. Transmission of Intestinal Bifidobacterium longum subsp. longum Strains from Mother to Infant, Determined by Multilocus Sequencing Typing and Amplified Fragment Length Polymorphism. Appl Environ Microbiol. 2011;77: 6788-93.

11. Mueller NT, Shin H, Pizoni A, Werlang IC, Matte U, Goldani MZ, et al. Birth mode-dependent association between pre-pregnancy maternal weight status and the neonatal intestinal microbiome. Sci. Rep. [Internet]. 2016 [cited 2016 Apr 12];6. Available from: http://www.ncbi.nlm.nih.gov/pmc/ articles/PMC4817027/.

12. Laursen MF, Andersen LBB, Michaelsen KF, Mølgaard C, Trolle E, Bahl MI, et al. Infant Gut Microbiota Development Is Driven by Transition to Family Foods Independent of Maternal Obesity. mSphere. 2016;1:e00069-15.

13. Galley JD, Bailey M, Kamp Dush C, Schoppe-Sullivan S, Christian LM Maternal Obesity Is Associated with Alterations in the Gut Microbiome in Toddlers. PLoS One. 2014;9:e113026.

14. Kalliomäki M, Collado MC, Salminen S, Isolauri E. Early differences in fecal microbiota composition in children may predict overweight. Am J Clin Nutr. 2008:87:534-8

15. White RA, Bjørnholt JV, Baird DD, Midtvedt T, Harris JR, Pagano M, et al. Novel Developmental Analyses Identify Longitudinal Patterns of Early Gut Microbiota that Affect Infant Growth. PLoS Comput Biol. 2013:9:e1003042.

16. Ajslev TA, Andersen CS, Gamborg M, Sørensen TIA, Jess T. Childhood overweight after establishment of the gut microbiota: the role of delivery mode, pre-pregnancy weight and early administration of antibiotics. Int J Obes. 2011;35:522-9.

17. Huh SY, Rifas-Shiman SL, Zera CA, Edwards JWR, Oken E, Weiss ST, et al Delivery by caesarean section and risk of obesity in preschool age children: a prospective cohort study. Arch Dis Child. 2012:97:610-6.

18. Center for Disease Control. Defining Adult Overweight and Obesity Overweight \& Obesity | CDC [Internet]. [cited 2016 May 5]. Available from: http://www.cdc.gov/obesity/adult/defining.html.

19. Weight Gain During Pregnancy: Reexamining the Guidelines [Internet]. National Academies Press; 2009. Available from: http://www. nationalacademies.org/hmd/Reports/2009/Weight-Gain-During-PregnancyReexamining-the-Guidelines.aspx.

20. Eggesbo M, Moen B, Peddada S, Baird D, Rugtveit J, Midtvedt T, et al. Development of gut microbiota in infants not exposed to medical interventions. APMIS Acta Pathol Microbiol Immunol Scand. 2011;119:17-35.

21. Edgar RC. Search and clustering orders of magnitude faster than BLAST. Bioinformatics. 2010;26:2460-1. 
22. Caporaso JG, Kuczynski J, Stombaugh J, Bittinger K, Bushman FD, Costello EK, et al. QIIME allows analysis of high-throughput community sequencing data. Nat Methods. 2010;7:335-6.

23. DeSantis TZ, Hugenholtz P, Larsen N, Rojas M, Brodie EL, Keller K, et al. Greengenes, a Chimera-Checked 165 rRNA Gene Database and Workbench Compatible with ARB. Appl Environ Microbiol. 2006;72:5069-72.

24. Lozupone C, Lladser ME, Knights D, Stombaugh J, Knight R. UniFrac: an effective distance metric for microbial community comparison. ISME J. 2011;5:169.

25. Breiman L. Random forests. Mach Learn. 2001;45:5-32.

26. Diaz-Uriarte RV. Variable selection using random forests. R Package Version 073 [Internet]. 2005 [cited 2016 Oct 13]; Available from: http://ftp.auckland. ac.nz/software/CRAN/doc/packages/varSelRF.pdf.

27. Knights D, Costello EK, Knight R. Supervised classification of human microbiota. FEMS Microbiol Rev. 2011;35:343-59.

28. R Core Team. R: The R Project for Statistical Computing. Vienna: R Foundation for Statistical Computing; 2015. [Internet]. [cited 2016 May 19]. Available from: https://www.r-project.org/.

29. Human Microbiome Project Consortium. Structure, function and diversity of the healthy human microbiome. Nature. 2012;486:207-14.

30. Lozupone CA, Stombaugh Jl, Gordon Jl, Jansson JK, Knight R. Diversity, stability and resilience of the human gut microbiota. Nature. 2012;489:220-30.

31. Mika A, Treuren W, González A, Herrera JJ, Knight R, Fleshner M. Exercise Is More Effective at Altering Gut Microbial Composition and Producing Stable Changes in Lean Mass in Juvenile versus Adult Male F344 Rats. PLoS One. 2015;10:e0125889.

32. Ridaura VK, Faith JJ, Rey FE, Cheng J, Duncan AE, Kau AL, et al. Gut Microbiota from Twins Discordant for Obesity Modulate Metabolism in Mice. Science. 2013:341:1241214

33. Verdam FJ, Fuentes $S$, de Jonge C, Zoetendal EG, Erbil R, Greve JW, et al. Human intestinal microbiota composition is associated with local and systemic inflammation in obesity. Obesity. 2013;21:E607-15.

34. Zhang H, DiBaise JK, Zuccolo A, Kudrna D, Braidotti M, Yu Y, et al. Human gut microbiota in obesity and after gastric bypass. Proc Natl Acad Sci. 2009; 106:2365-70.

35. Walters WA, Xu Z, Knight R. Meta-analyses of human gut microbes associated with obesity and IBD. FEBS Lett. 2014;588:4223-33.

36. Goodrich JK, Waters JL, Poole AC, Sutter JL, Koren O, Blekhman R, et al. Human Genetics Shape the Gut Microbiome. Cell. 2014;159:789-99.

37. Schwiertz A, Taras D, Schäfer K, Beijer S, Bos NA, Donus C, et al. Microbiota and SCFA in Lean and Overweight Healthy Subjects. Obesity. 2010;18:190-5.

38. Santacruz A, Collado MC, García-Valdés L, Segura MT, Martín-Lagos JA, Anjos T, et al. Gut microbiota composition is associated with body weight, weight gain and biochemical parameters in pregnant women. Br J Nutr. 2010;104:83-92.

39. Sze MA, Schloss PD. Looking for a Signal in the Noise: Revisiting Obesity and the Microbiome. MBio. 2016;7:e01018-6.

40. Ege MJ, Mayer M, Normand A-C, Genuneit J, Cookson WOCM, BraunFahrländer C, et al. Exposure to Environmental Microorganisms and Childhood Asthma. N Engl J Med. 2011;364:701-9.

41. Koren O, Goodrich JK, Cullender TC, Spor A, Laitinen K, Kling Bäckhed H, et al. Host Remodeling of the Gut Microbiome and Metabolic Changes during Pregnancy. Cell. 2012;150:470-80.

42. Arrieta M-C, Stiemsma LT, Dimitriu PA, Thorson L, Russell S, Yurist-Doutsch S, et al. Early infancy microbial and metabolic alterations affect risk of childhood asthma. Sci Transl Med. 2015;7:307ra152-307ra152.

43. Le Chatelier E, Nielsen T, Qin J, Prifti E, Hildebrand F, Falony G, et al. Richness of human gut microbiome correlates with metabolic markers. Nature. 2013:500:541-6.

44. Kang D-W, Park JG, Ilhan ZE, Wallstrom G, LaBaer J, Adams JB, et al. Reduced Incidence of Prevotella and Other Fermenters in Intestinal Microflora of Autistic Children. PLoS One. 2013;8:e68322.

45. DiGiulio DB, Callahan BJ, McMurdie PJ, Costello EK, Lyell DJ, Robaczewska A, et al. Temporal and spatial variation of the human microbiota during pregnancy. Proc Natl Acad Sci. 2015;112:11060-5.

46. Egshatyan L, Kashtanova D, Popenko A, Tkacheva O, Tyakht A, Alexeev D, et al. Gut microbiota and diet in patients with different glucose tolerance. Endocr Connect. 2015;5:1-9.

47. Kasai C, Sugimoto K, Moritani I, Tanaka J, Oya Y, Inoue H, et al. Comparison of the gut microbiota composition between obese and non-obese individuals in a Japanese population, as analyzed by terminal restriction fragment length polymorphism and next-generation sequencing. BMC Gastroenterol. 2015;15:100.
48. Jeng RR, Taur Y, Devlin SM, Ponce DM, Goldberg JD, Ahr KF, et al. Intestinal Blautia Is Associated with Reduced Death from Graft-versus-Host Disease. Biol Blood Marrow Transplant. 2015;21:1373-83.

49. Bokulich NA, Chung J, Battaglia T, Henderson N, Jay M, Li H, et al. Antibiotics, birth mode, and diet shape microbiome maturation during early life. Sci Transl Med. 2016;8:343ra82-343ra82.

50. Collado MC, Isolauri E, Laitinen K, Salminen S. Effect of mother's weight on infant's microbiota acquisition, composition, and activity during early infancy: a prospective follow-up study initiated in early pregnancy. Am J Clin Nutr. 2010;92:1023-30.

51. Nayfach S, Rodriguez-Mueller B, Garud N, Pollard KS. An integrated metagenomics pipeline for strain profiling reveals novel patterns of bacterial transmission and biogeography. Genome Res. 2016;26:1612-25.

52. Barker D. The developmental origins of chronic adult disease. Acta Paediatr. 2004;93:26-33.

53. Mueller NT, Bakacs E, Combellick J, Grigoryan Z, Dominguez-Bello MG. The infant microbiome development: mom matters. Trends Mol Med. 2015;21:109-17.

54. Mitchell A, Bucchini F, Cochrane G, Denise H, ten Hoopen P, Fraser M, et al. EBI metagenomics in 2016 - an expanding and evolving resource for the analysis and archiving of metagenomic data. Nucleic Acids Res. 2016;44: D595-603.

\section{Submit your next manuscript to BioMed Central and we will help you at every step:}

- We accept pre-submission inquiries

- Our selector tool helps you to find the most relevant journal

- We provide round the clock customer support

- Convenient online submission

- Thorough peer review

- Inclusion in PubMed and all major indexing services

- Maximum visibility for your research

Submit your manuscript at www.biomedcentral.com/submit

) Biomed Central 